\title{
Whole-exome sequencing on deceased fetuses with ultrasound anomalies: expanding our knowledge of genetic disease during fetal development
}

\author{
Carin L. Yates, MS, Kristin G. Monaghan, PhD, Deborah Copenheaver, MS, Kyle Retterer, MS, \\ Julie Scuffins, MS, Cathlin R. Kucera, MS, Bethany Friedman, MS, Gabriele Richard, MD and \\ Jane Juusola, PhD
}

Purpose: The aim of this study was to determine the diagnostic yield of whole-exome sequencing (WES) in fetuses with ultrasound anomalies that resulted in fetal demise or pregnancy termination. The results were also utilized to aid in the identification of candidate genes for fetal development and to expand the clinical phenotype of known genetic conditions.

Methods: WES was performed on specimens from 84 deceased fetuses. Data were analyzed and final results were classified into one of four categories: positive, possible, negative, and candidate gene only. WES analysis was predominantly performed in fetus-parent trios or quads $(61 \%, \mathrm{n}=52)$.

Results: Overall, $20 \%(n=17)$ of cases were positive, $45 \%$ $(n=38)$ were possible, $9 \%(n=7)$ had only candidate gene variants and 26\% $(n=22)$ tested negative. The diagnostic yield for definitive findings for trio analysis was $24 \%(n=11)$ compared to $14 \%(n=4)$ for singletons. The most frequently reported ultrasound anomalies were central nervous system $(37 \%, n=31)$, hydrops/edema $(36 \%, n=30)$, and cardiovascular anomalies $(31 \%, n=26)$.

Conclusion: Our experience supports the use of WES to identify the molecular etiology of fetal ultrasound anomalies, to identify candidate genes involved in fetal development, and to expand our knowledge of the clinical phenotype of known genetic conditions.

Genet Med advance online publication 20 April 2017

Key Words: exome; fetal; ultrasound anomaly; WES

\section{INTRODUCTION}

Congenital malformations are identified in approximately $3 \%$ of live births in the United States, accounting for one-third of perinatal deaths and contributing to $20 \%$ of infant morbidity. ${ }^{1,2}$ When including structural fetal anomalies identified during pregnancy, the overall prevalence of fetal malformations is likely higher due to a proportion of these pregnancies that are miscarried, stillborn, or terminated. Chromosome anomalies, single- or multiple-gene disorders, and multifactorial conditions have long been recognized as major contributors to birth defects, although the etiology of many congenital malformations is unknown. ${ }^{3}$

The identification of a fetal ultrasound anomaly prompts additional prenatal evaluations. Fetal specimens obtained through invasive testing such as chorionic villus sampling or amniocentesis can be utilized for genetic testing. Array-based comparative genomic hybridization can detect chromosome aneuploidies, unbalanced translocations, and copy number variants that are cytogenetically cryptic by standard G- banded chromosome analysis. The diagnostic yield for array-based comparative genomic hybridization is approximately $6 \%$ higher than conventional karyotyping in fetuses with structural anomalies identified by ultrasound. ${ }^{4,5}$ For fetal ultrasound anomalies suggestive of a specific disorder, single- gene testing and gene panels with a small number of genes can be utilized as an initial step in molecular diagnosis. However, the etiology for many fetal ultrasound anomalies remains unidentified despite the increased diagnostic rate of array-based comparative genomic hybridization and the availability of single-gene tests and gene panels for some of the most common genetic disorders with known fetal manifestation. Accurate genetic counseling regarding the prognosis for the fetus and recurrence risks in future pregnancies is usually not possible without a definitive diagnosis.

Whole-exome sequencing (WES) has been utilized in pediatric and adult clinical practice to identify the underlying genetic cause of disease when prior testing has failed to provide a diagnosis. WES is increasingly considered as a firsttier molecular test with increased diagnostic and clinical utility over standard investigations. ${ }^{6,7}$ Additionally, WES has enabled the discovery of many new genotype-phenotype associations; for example, pathogenic variants in the TKT gene are found in individuals with short stature, developmental delay, and congenital heart defects; and pathogenic variants in the CTBP1 gene have been shown to cause developmental delay, hypotonia, ataxia, and tooth enamel defects. ${ }^{89}$ Using WES in pediatric and adult populations, we 
previously demonstrated in a large clinical series an overall diagnostic yield of $28.8 \%$ with a range of $24 \%$ for singleton cases to $31 \%$ for trio-based analysis, which is comparable to other laboratories reporting yields of 22 to $26 \% .^{10-13}$

Fetal specimens submitted for exome sequencing to investigate ultrasound anomalies comprise a unique phenotypic cohort. Previous studies of fetuses with ultrasound abnormalities reported a wide range in diagnostic yield for WES, from 10 to $57 \%$, but were hampered by their small sample size. ${ }^{12-17}$ As a clinical diagnostic laboratory, we have analyzed the WES results of 84 specimens collected from a deceased fetus. These results have improved our understanding of the diagnostic yield of WES for fetal ultrasound anomalies, expanded the clinical phenotype of known genetic conditions, and assisted in the identification of candidate genes for fetal anomalies. To our knowledge, this is the largest series of deceased fetal WES cases reported to date.

\section{MATERIALS AND METHODS}

We reviewed 84 fetal WES cases from pregnancies with ultrasound anomalies that were terminated or resulted in fetal demise between October 2012 and March 2016. WES was performed using methods as previously described on genomic DNA from the deceased fetus and any submitted family members. ${ }^{10}$

Fetal DNA was submitted directly in $63 \%(N=53)$ of cases. In the remaining cases, fetal DNA was obtained from various submitted sources, including cultured amniocytes $(11 \%, N=9)$, cord blood from a fetal demise or at the time of termination via induction $(9.5 \%, \mathrm{~N}=8)$, cultured cells from products of conception $(9.5 \%, \mathrm{~N}=8)$, direct products of conception (5\%, N $=4$ ), and direct amniotic fluid $(2 \%, N=2)$. Parental DNA was obtained from peripheral blood or oral rinse. Clinicians were encouraged to provide blood or DNA specimens for both parents and DNA from previously affected pregnancies when available. Reported variants were confirmed by dideoxy sequencing. No copy number variants were identified in this cohort. Maternity and paternity were confirmed by kinship analysis of the WES data using KING. ${ }^{18}$

The fetuses had a range of anomalies identified by prenatal imaging, post-mortem examination, or autopsy. The phenotypic information and differential diagnoses provided by the referring physicians were converted to Human Phenotype Ontology, Human Gene Mutation Database, and Online Mendelian Inheritance in Man database terms and used for WES analysis as previously described. ${ }^{10}$ As of 30 September 2015, the American College of Medical Genetics and Genomics (ACMG) variant classification recommendations were utilized for all reported variants. ${ }^{19}$ Although more than one result may have been reported for a case, the variant with the highest classification was used to categorize the overall results. Secondary findings were investigated as recommended from the ACMG list of 56 designated genes beginning May 2013 unless the family opted out of receiving this information. ${ }^{20}$ The presence of secondary findings was not considered in the overall classification of the case and a case in any of the categories below may have had a secondary finding reported. The overall case was classified into one of the four categories below:

- Positive result: pathogenic or variant(s) likely pathogenic in a known disease gene associated with the reported phenotype.

- Possible diagnosis: variant(s) in a known disease gene possibly associated with the reported phenotype. This category includes novel variants, including missense variants or in-frame insertions/deletions in disease genes, that overlap the phenotype provided for the proband. This category also includes recessive conditions that overlap with the phenotype provided for the proband in which only a single pathogenic variant is identified.

- Candidate gene: variant(s) predicted to be deleterious in a novel candidate gene that have not previously been implicated in human disease or for which the published data to support human disease association may not yet be definitive. Supporting data could be based on model organism data, copy number variant data, tolerance of the gene to sequence variation, data about tissue or developmental timing of expression, or knowledge of the gene function and pathway analysis. Further research is required to evaluate any of the suggested candidate genes.

- Negative result: no variants in genes associated with the reported phenotype identified.

\section{RESULTS}

We analyzed results from WES in 84 deceased fetuses, with 29 (34\%) submitted as fetal singleton only, $4(5 \%)$ as maternalfetal duos, 45 (54\%) as traditional proband-parent trios, and six (7\%) quads comprised of both parental and sibling samples. All six of the quads were from families with previously affected pregnancies. Sibling samples in two cases came from a prior affected pregnancy, one from an affected living sibling, and three were from unaffected, living siblings where DNA from the prior affected pregnancy was not available for testing. Previously affected pregnancies were reported in $27(32 \%)$ cases, $24(89 \%)$ with a similar phenotype, and three (11\%) with a discordant phenotype. Gestational age of the fetus was reported in $49(58 \%)$ cases; $30(61 \%)$ were in the second trimester and $19(39 \%)$ in the third trimester. The mean gestational age was 24 weeks, with a range from 14 to 39 weeks. However, it was not always apparent if the reported gestational age represented the time of diagnosis, invasive procedure, fetal demise, or termination. Of the 40 cases that reported specific information regarding fetal outcome, $38 \%(15 / 40)$ ended in pregnancy termination and $62 \%(25 / 40)$ resulted in fetal demise/stillbirth. Cases of fetal demise or stillbirth without ultrasound anomalies were not included in this series. Fetal sex as determined by WES data was $55 \%(\mathrm{~N}=46)$ male and $45 \%(\mathrm{~N}=38)$ female. Three cases had discordant genetic sex from phenotypic sex: 46,XX 
with a male phenotype, 46,XY with a female phenotype, and $46, \mathrm{XY}$ with ambiguous genitalia.

Prior karyotype and/or microarray analysis was reported in $68(80 \%)$ fetuses. Of those, 46 (68\%) had both karyotype and microarray analysis, four (6\%) had karyotype only, and 18 (26\%) had microarray analysis only. All results were normal except in two cases in which paternally inherited, nondiagnostic microdeletions were found. Prior single-gene or panel testing for a wide range of genetic conditions was reported in 14 (17\%) cases, most commonly panels for Noonan syndrome, fetal akinesia deformation sequence, hydrops, and congenital nephrotic syndrome. Prior gene testing was negative for all cases. Clinical information provided for each case was variable and included consultation notes, ultrasound reports, fetal magnetic resonance imaging and echocardiogram results, postmortem examination, and fetal autopsy.

Of the 84 cases analyzed, 17 cases (20\%) yielded a definitive diagnosis. Of the remaining cases, variant(s) with a possible relationship to phenotype were identified in 38 cases (45\%), 22 cases $(26 \%)$ were reported as negative, and a candidate gene was the only finding in seven (9\%) cases. For cases submitted as trios, $24 \%(11 / 45)$ had a positive result versus $14 \%(4 / 29)$ positive in singleton cases. Of the 27 cases with previously affected pregnancies, three (11\%) were positive, 11 (41\%) had possible results, two (7\%) had candidate gene-only results, and 11 (41\%) were negative. Although our sample size is small, the positive yield was $11 \%(3 / 27)$ in cases with a prior affected pregnancy and $25 \%(14 / 57)$ in cases with no reported prior affected pregnancy. Of the cases with prior microarray testing, 23\% (15/64) had a positive WES result. Of the cases without identifiable chromosomal aberrations on prior cytogenetic analysis (karyotype and/or microarray testing), $24 \%(16 / 68)$ had a positive WES result. In the entire cohort, two cases had ACMG secondary findings in genes associated with cardiac arrhythmia. A $\mathrm{KCNH} 2$ pathogenic variant was reported in a case where the familial variant had been previously identified in the fetus through prenatal testing by an outside laboratory and an inherited $D S P$ pathogenic variant was reported as a secondary finding in another case. The variants in $\mathrm{KCNH} 2$ and DSP were not related to the presenting fetal phenotype and were reported in accordance with ACMG recommendations for secondary findings in WES. $^{20}$ Table 1 lists the frequency of each category of ultrasound anomalies in the entire cohort, with central nervous system, hydrops/edema, and cardiovascular anomalies the three most frequent findings overall. To illustrate the diversity of ultrasound findings reported, examples of anomalies in each category have been listed in Table 1 . Of the entire cohort, 52 cases (62\%) presented with multiple congenital anomalies. The remaining 32 cases $(38 \%)$ presented with an isolated ultrasound anomaly. The diagnostic yield was almost identical in the group with multiple congenital anomalies as the group with an isolated anomaly: $20 \%(10 / 52)$ and $21 \%(7 / 32)$, respectively. The distribution of clinical indications for cases with a positive diagnostic finding was similar to those in the entire cohort (Figure 1).

The 17 cases with positive results are listed in Table 2. In one of these cases, we initially reported two pathogenic variants, a de novo PIK3CA variant associated with several segmental overgrowth conditions and an inherited $\mathrm{MYH3}$ variant predicted to cause arthrogryposis. When applying the new, more conservative ACMG variant classification criteria, the MYH3 variant was reclassified as likely benign based on allele frequency data in presumed healthy control populations. ${ }^{19}$ One de novo variant in the PTPN11 gene was categorized as a "possible" result as the contribution of this pathogenic variant to the overall fetal phenotype was unclear and a pathogenic variant had also been reported in the FLNA gene. When evaluating the inheritance patterns of the reported variants in the positive cases, we found seven (41\%) cases with dominant inheritance, five of which were de novo; four (24\%) with recessive inheritance; five (29\%) with $\mathrm{X}$-linked inheritance, one of which was de novo; and one (6\%) with two variants in the PIEZO1 gene, which has been associated with both autosomal dominant and recessive generalized lymphatic dysplasia. Forty-one percent (7/17) of

Table 1 Frequency of anomalies identified and examples of specific findings

\begin{tabular}{|c|c|c|}
\hline Category of anomaly & Frequency of anomaly & Examples of specific findings \\
\hline Central nervous system & $37 \%(N=31)$ & $\begin{array}{l}\text { Structural anomalies, ventriculomegaly/hydrocephalus, neural migration disorders, } \\
\text { hypoplasia }\end{array}$ \\
\hline Hydrops/edema & $36 \%(N=30)$ & Hydrops, edema, cystic hygroma, ascites \\
\hline Cardiovascular system & $31 \%(N=26)$ & Complex congenital heart defect, hypoplastic left heart, cardiomegaly, cardiomyopathy \\
\hline Genitourinary system & $27 \%(N=23)$ & Renal cysts, renal agenesis, ambiguous genitalia, pyelectasis/hydronephrosis \\
\hline Skeletal system & $24 \%(N=20)$ & Syndactyly, polydactyly, bowed long bones, kyphoscoliosis, absent and hypoplastic radius/ulna \\
\hline Respiratory system & $11 \%(N=9)$ & Diaphragmatic hernia; lung hypoplasia \\
\hline Growth abnormality & $10 \%(N=8)$ & Intrauterine growth restriction \\
\hline Gastrointestinal system & $6 \%(N=5)$ & Absent stomach, small stomach \\
\hline
\end{tabular}

Boldfaced ultrasound anomalies were seen as both isolated findings and in cases with multiple congenital anomalies. The remaining ultrasound findings were identified only in cases with multiple congenital anomalies. 


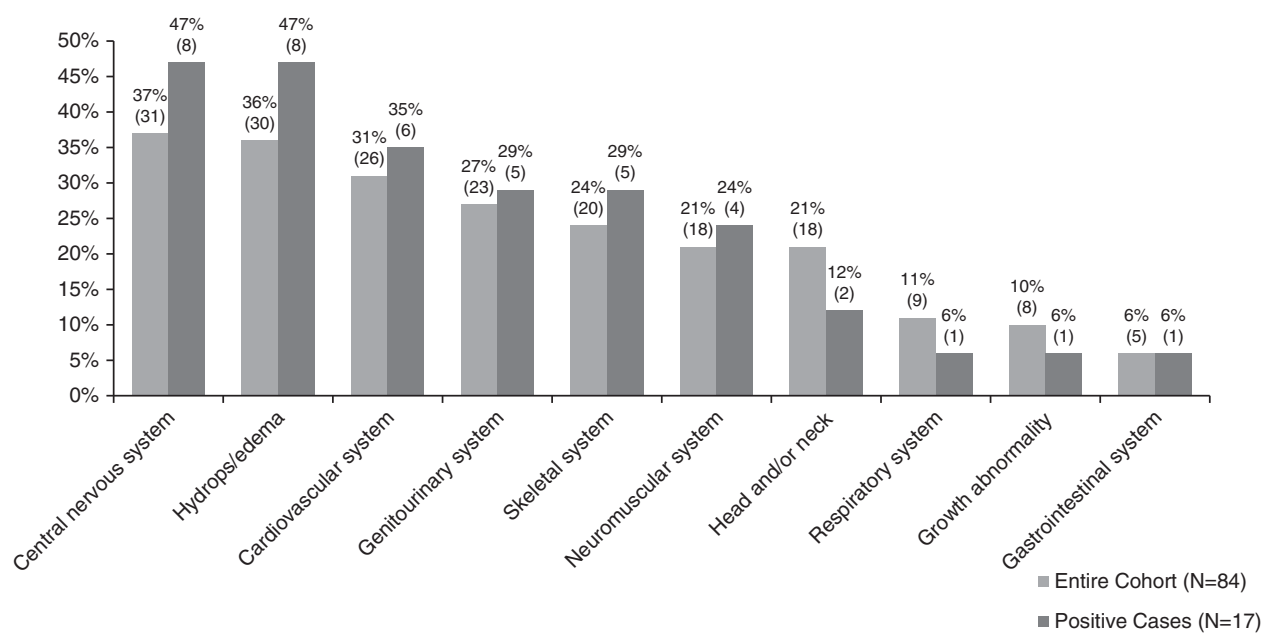

Figure 1 Frequency of ultrasound anomalies in the entire cohort compared to the positive cases only. The number of cases ( $\mathrm{N}$ ) for each category is shown in parentheses following the percentage.

the positive cases had a reported de novo variant; six reported as a positive result and one as a possible result. In our cohort, $15 \%(13 / 84)$ had variants in candidate genes as shown in Table 3 ; seven cases had only candidate gene variants reported and six of these cases had a positive or possible result in addition to the variant(s) in the candidate gene. All reported variants in our cohort are listed in Supplementary Table 1.

\section{DISCUSSION}

In our cohort of 84 fetal WES cases, which is the largest series reported to date, we observed positive diagnostic findings in $20 \%$ of cases. Several smaller series on fetal WES have reported positive results in $10-57 \%$ of cases. Specifically, in two consecutive studies, Yang and colleagues identified positive results in 7 of 15 deceased fetuses. ${ }^{12,13}$ In both studies, all cases had some prior genetic testing and were clinically classified into one of four primary phenotypes based on the presence or absence of neurologic disorders and any additional organ involvement. Clinical information on the fetal specimens was limited to more general categories such as "neurologic disorder and other organ-system disorder" and "nonneurologic disorder." ${ }^{2,13}$ As these cases were part of larger studies of primarily pediatric and adult patients with limited phenotypic information for the fetal specimens, it is difficult to establish if these 15 fetal cases are comparable to our cohort. Another study reported on 30 trio-based WES cases in fetuses and neonates with normal karyotype that presented with diverse ultrasound anomalies similar to our cohort and three of the $30(10 \%)$ had a definitive result. ${ }^{14}$ Drury et al. reported on 24 cytogenetically normal fetal WES cases with ultrasound anomalies similar to our study. ${ }^{15}$ In $21 \%(5 / 24)$ of these cases, a definitive diagnosis was found, consistent with our findings of $20 \% .{ }^{15}$ As part of a larger series of families considering WES in a reproductive genetic counseling practice, Westerfield et al. reported a diagnostic rate of $30 \%(3 / 10)$ for pregnancies with ultra- sound anomalies. ${ }^{16}$ In another small study, Alamillo et al. reported on seven fetal specimens from pregnancies with ultrasound anomalies; three had "positive" results and one had a "likely positive" result, for a detection rate of $43 \%(3 / 7)$ to $57 \%(4 / 7) .{ }^{17}$ The importance of analyzing trios to increase the diagnostic yield of WES has been well demonstrated in postnatal WES studies, and also applies to WES in a prenatal setting. ${ }^{10,21}$ In both our study and the one by Drury et al., $14 \%$ of singleton cases had a positive result and this number increased when trios were analyzed, to $24 \%$ in our study and $30 \%$ in the report by Drury et al. ${ }^{15}$ This gain in sensitivity is mostly due to the ability to identify de novo variants and determine phase for variants identified in recessive genes. ${ }^{10}$ Although little has been previously published on WES for fetal anomalies, our results of a $20 \%$ overall positive molecular diagnosis and increase in diagnostic yield for trio samples are consistent with prior studies of fetuses with diverse ultrasound anomalies $(p=0.4675)$.

Identifying an accurate fetal phenotype can be difficult. Detection rates for fetal anomalies in general are variable and may be dependent on the experience of the center, the type of imaging utilized, and the gestational age of the fetus. ${ }^{22,23} \mathrm{In}$ our cohort, there were also inconsistencies in the quality and quantity of clinical information provided. For example, some submissions had detailed clinical notes and imaging reports that gave very specific summaries of clinical findings whereas other cases may have had generic terms such as "heart defect" or "brain anomaly" with no further information provided. Our cohort also represents only a subpopulation of fetuses presenting with ultrasound anomalies as pregnancies that underwent prior genetic testing that provided a diagnosis would not be referred for WES. However, even with these limitations some general observations regarding the clinical indications can be made. The most frequent ultrasound anomalies in both the entire cohort and the positive cases included central nervous system anomalies, hydrops/edema, 
Table 2 Clinical phenotype and identified variants in positive cases

Primary molecular diagnosis

p.E679X (Homozygous PATH)

p.E679X (Homozygous PATH)
BBS4 (AR)

Disorder

Other reported variants

Disorder

Generalized lymphatic dysplasia $\quad$ PIEZO1 (AD/AR) Generalized

p.A1496V (Homozygous VUS) $\quad$ lymphatic dysplasia

Bilateral enlarged cystic kidneys $\quad$ C.1106+2T > A (Homozygous PATH)
a

Bardet-Biedl syndrome

ANKS6 (AR)

Nephronophthisis 16

p.D313N (Heterozygous VUS)

Polycystic kidney

PKD1 (AD)

disease

p.P4162S (Heterozygous VUS)

HRAS (AD) Costello syndrome

p.G13D (Heterozygous PATH) ${ }^{a}$

Hydrops, diaphragmatic hernia, gracile ribs, contractures RIPK4 (AR)

p.A10S (Heterozygous LPATH) ${ }^{a}$

p.V303M (Heterozygous VUS)

Bartsocas-Papas syndrome

RSAD1 (unknown)

p.W431X (Homozygous VUS)

Unknown

FGFR2 (AD)

Frontal bossing, talipes, syndactyly, abducted thumbs

c.940-1G > A (Heterozygous PATH)

PTPN11 (AD)

p.N58K (Heterozygous PATH) ${ }^{\text {a }}$

PIK3CA (AD)

p.E545K (Heterozygous PATH) ${ }^{a}$

Brain malformations

FOXP3 (XL)

Hydrops, contractures, echogenic kidney,

placentalmegaly

Hydrops, CNS malformations, cardiomyopathy

p.R337X (Hemizygous PATH)

MRPS22 (AR)

c.768_769del (Heterozygous PATH)

p.R170H (Heterozygous PATH) ${ }^{\mathrm{a}}$

CNS malformations

FLNA (XL)

p.V552I (Hemizygous LPATH)

Hydrops, cardiomegaly

CYP11A1 (AR)

p.R120X (Homozygous PATH)

Ventriculomegaly, cardiac left-axis deviation, absent radii FANCB (XL)

c.987_990del (Hemizygous PATH)

AMER1 (XL) Osteopathia striata with cranial sclerosis

Macrocephaly, cleft lip and palate, congenital heart

c.705delT (Hemizygous PATH)

RIT1 (AD)

p.F82C (Heterozygous PATH)

Hydrops, CNS malformation, congenital heart defect

Megalencephaly, neuronal migrational anomaly,

congenital heart defect, heterotopias

PIK3R2 (AD)

PPAP2C (unknown) p.R214Q (Heterozygous VUS)

FGFR2-related disorder

Noonan syndrome

WDR35 (AR)

p.G103V (Heterozygous LPATH)

Cranioectodermal

PIK3CA-related overgrowth syndromes

MYH3 (AD)

dysplasia

p.A1637V (Heterozygous PATH; reclassified to LBEN)

COL10A1 (AD)

c.1632delG (Heterozygous PATH)

Schmid metaphyseal

MRPS22-related mitochondria

chondrodysplasia

dysfunction

FLNA-related disorder

PTPN11 (AD)

Noonan syndrome

Adrenal insufficiency

\section{p.D61G (Heterozygous PATH)}

Confirmed de novo variants are shown in bold.

apositive result.

bPossible result.
${ }^{c}$ Candidate gene.

p.K564E (Heterozygous LPATH)

$\operatorname{LICAM}(\mathrm{XL})$

(Hemizygous PATH)

c.738delG (Heterozygous PATH)

Megalencephaly-polymicrogyria-

polydactyly-hydrocephalus

Hydrocephalus

Campomelic dysplasia 
Table 3 Variants reported in genes classified as candidate genes

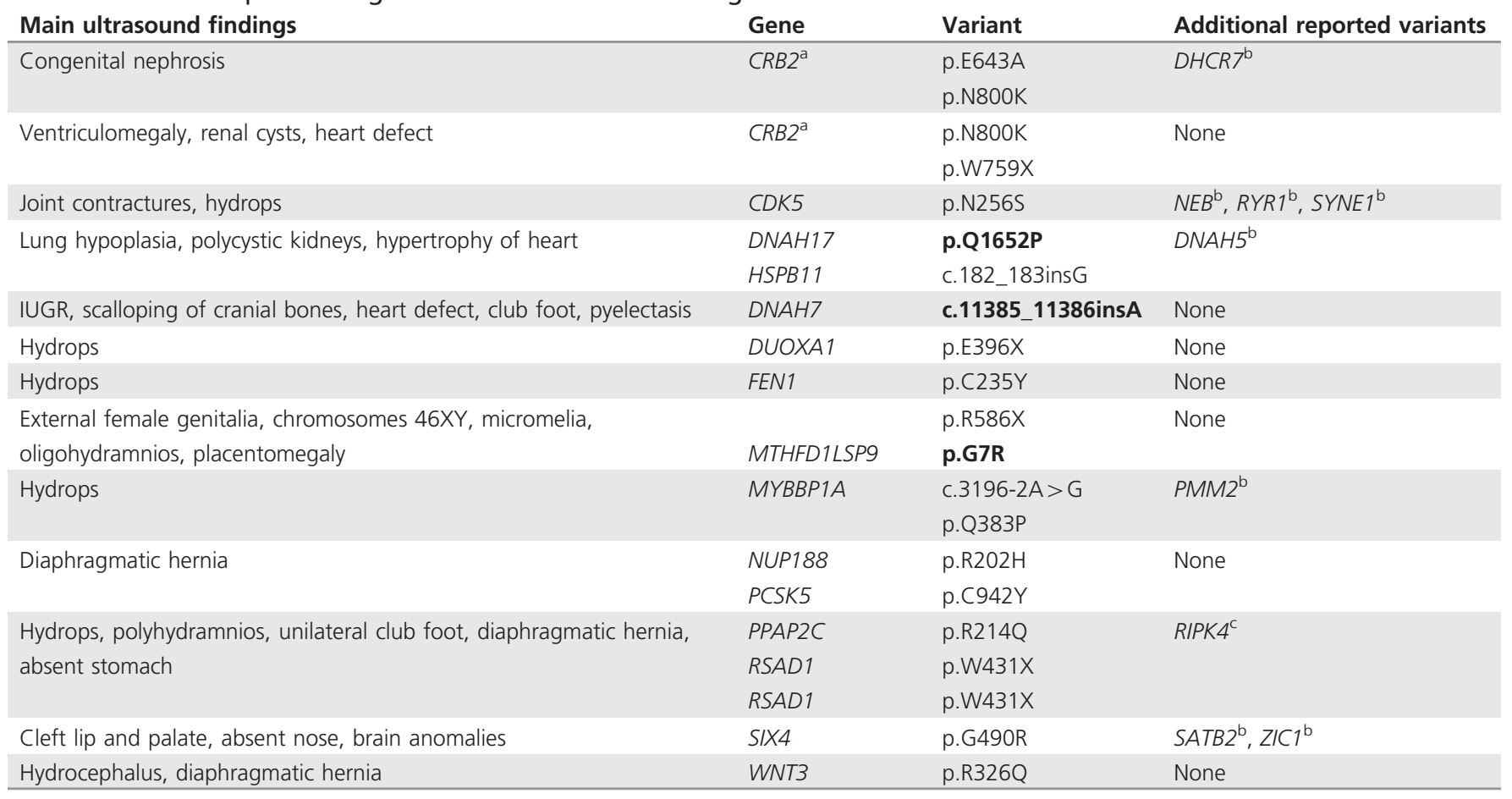

Confirmed de novo variants shown in bold.

${ }^{a}$ Gene now published and no longer a candidate gene; known cause of recessive nephritic syndrome.

${ }^{\mathrm{b}}$ Possible result.

'Positive result.

and cardiovascular anomalies (Figure 1). The positive diagnostic yield was similar for the fetuses that presented with multiple congenital anomalies $(19 \%, 10 / 52)$ compared to those that presented with a single ultrasound anomaly $(21 \%, 7 / 32)$. Although our data are limited by a small sample size and scarcity of detailed information on the ultrasound findings themselves, our results suggest that WES could be considered a diagnostic option for a wide spectrum of fetal ultrasound anomalies when standard testing, such as fetal karyotype and/ or microarray, has failed to provide a diagnosis.

An early definitive diagnosis for a fetus with ultrasound abnormalities allows for more accurate prognostic predictions, establishing an appropriate delivery strategy, a pre- and postnatal management plan, as well as better recurrence risk assessment in the family. In a subset of our cohort, we identified variants in genes known to manifest prenatally and the molecular diagnosis was consistent with the ultrasound findings. For example, one fetus with macrocephaly, cleft lip and palate, congenital heart defect, bifid thumb, and hydrocephalus had an inherited pathogenic variant in the AMER1 gene, which is associated with X-linked osteopathia striata with cranial sclerosis. The parent from which the variant was inherited had reportedly minor dysmorphic features and there was a previously affected pregnancy. The clinical features of our case were consistent with previously reported prenatal cases. ${ }^{24,25}$ Other examples of cases with pathogenic variants in genes with known prenatal manifestations include PTPN11 associated with Noonan syndrome, L1CAM associated with hydrocephalus, and SOX9 associated with campomelic dysplasia.

Nevertheless, data from fetal WES can also provide new insights in the spectrum and frequency of early developmental abnormalities in novel disorders or for well-established genetic conditions without known prenatal involvement. For example, one case in our series was a fetus that presented with hydrops in the third trimester resulting in fetal demise. The pregnancy history was significant for a prior pregnancy with the same presentation and outcome. WES on the fetus identified compound heterozygous variants of uncertain significance in FZD6, a gene associated with autosomal recessive nonsyndromic congenital nail disorder 10 and an increased risk of neural tube defects. ${ }^{26,27}$ While at the time of analysis an association between FDZ6 variants and hydrops was unknown, Shamseldin et al. recently reported a homozygous FDZ6 variant associated with fetal hydrops in a study to identify embryonic lethal genes. ${ }^{28}$ Another case in our series was diagnosed with megalencephaly-polymicrogyriapolydactyly-hydrocephalus syndrome after a de novo likely pathogenic variant in the PIK3R2 gene was identified on fetal WES. The fetus had presented prenatally with a complex heart defect and dilated ventricle and the pregnancy resulted in a stillbirth at 20 weeks. PIK3R2 is associated with several conditions of segmental overgrowth conditions, with limited information on its prenatal presentation. 
Fetal WES is also a useful tool in identifying novel candidate genes that have not previously been implicated in human disease. For example, we identified in our series two cases presenting with renal cysts who were compound heterozygous for two missense variants in the CRB2 gene. At the time of initial analysis and reporting, this gene was considered a candidate gene and consequently the specific variants were considered variants of uncertain clinical significance. Subsequently, both of these cases were published as part of a series of five fetuses and a male infant who died at 7 months of age from three families with pathogenic variants in CRB2 causing an newly described disorder: autosomal recessive nephrotic syndrome associated with ventriculomegaly. ${ }^{29-31}$ By analyzing the entire fetal exome, WES allows for a broader search of disease-causing genes compared to focused multigene panel tests, including those that would not typically be considered due to limited known information during fetal development. These cases help to expand our knowledge of the prenatal presentation of known genetic conditions and identify candidate genes that are involved in fetal development.

The overall clinical utility of WES for fetal ultrasound anomalies is still being elucidated. Understanding the benefits and limitations of this new technology is imperative to ensure appropriate clinical management. ${ }^{32,33}$ The American College of Obstetrics and Gynecology recommends microarray as the primary genetic test for pregnancies presenting with fetal ultrasound anomalies. ${ }^{34}$ However, WES for fetal ultrasound anomalies will continue to evolve as our knowledge increases and technology improves. The prenatal presentation of most single-gene genetic conditions is currently unknown and will advance with the continuing expansion of the phenotype of genetic disease. ${ }^{35}$ In addition, the variant filtering process continues to improve, as does the standardization of variant classification across laboratories and the resources for public sharing of data, such as ClinVar. ${ }^{19,36,37}$ All of these factors play a role in the importance of re-evaluating cases over time, as the accumulation of knowledge will contribute toward improving the diagnostic yield of WES in the perinatal setting and expanding our knowledge of the prenatal presentation of known genetic conditions, as well as aid in identifying candidate genes.

\section{SUPPLEMENTARY MATERIAL}

Supplementary material is linked to the online version of the paper at http://www.nature.com/gim

\section{DISCLOSURE}

All of the authors are employees of GeneDx.

\section{REFERENCES}

1. Centers for Disease Control and Prevention. Update on overall prevalence of major birth defects-Atlanta, Georgia, 1978-2005. MMWR Morb Mortal Wkly Rep 2008;57:1-5.

2. Matthews TJ, MacDorman MF, Thoma ME. Infant mortality statistics from the 2013 period linked birth/infant death data set. Natl Vital Stat Rep $2015 ; 64: 1-30$
3. Baird PA, Anderson TW, Newcombe HB, Lowry RB. Genetic disorders in children and young adults: a population study. Am J Hum Genet 1988;42:677-693.

4. Wapner RJ, Martin CL, Levy B, et al. Chromosomal microarray versus karyotyping for prenatal diagnosis. N Engl J Med 2012;367: 2175-2184.

5. Shaffer LG, Dabell MP, Fisher AJ, et al. Experience with microarray-based comparative genomic hybridization for prenatal diagnosis in over 5000 pregnancies. Prenat Diagn 2012;32:976-985.

6. Xue Y, Ankala A, Wilcox WR, Hegde MR. Solving the molecular diagnostic testing conundrum for Mendelian disorders in the era of next-generation sequencing: single-gene, gene panel, or exome/genome sequencing. Genet Med 2015;17:444-451.

7. Stark Z, Tan TY, Chong B, et al. A prospective evaluation of whole-exome sequencing as a first-tier molecular test in infants with suspected monogenic disorders. Genet Med 2016;18:1090-1096.

8. Boyle L, Wamelink MM, Salomons GS, et al. Mutations in TKT are the cause of a syndrome including short stature, developmental delay, and congenital heart defects. Am J Hum Genet 2016;98:1235-1242.

9. Beck DB, Cho MT, Millan F, et al. A recurrent de novo CTBP1 mutation is associated with developmental delay, hypotonia, ataxia, and tooth enamel defects. Neurogenetics 2016;17:173-178.

10. Retterer K, Juusola J, Cho MT, et al. Clinical application of whole-exome sequencing across clinical indications. Genet Med 2016;18:696-704.

11. Lee $H$, Deignan JL, Dorrani N, et al. Clinical exome sequencing for genetic identification of rare Mendelian disorders. JAMA 2014;312:1880-1887.

12. Yang Y, Muzny DM, Reid JG, et al. Clinical whole-exome sequencing for the diagnosis of mendelian disorders. N Engl J Med 2013;369: 1502-1511.

13. Yang $Y$, Muzny DM, Xia F, et al. Molecular findings among patients referred for clinical whole-exome sequencing. JAMA 2014;312:1870-1879.

14. Carss KJ, Hillman SC, Parthiban V, et al. Exome sequencing improves genetic diagnosis of structural fetal abnormalities revealed by ultrasound. Hum Mol Genet 2014;23:3269-3277.

15. Drury $S$, Williams $H$, Trump $N$, et al. Exome sequencing for prenatal diagnosis of fetuses with sonographic abnormalities. Prenat Diagn 2015;35:1010-1017.

16. Westerfield LE, Stover SR, Mathur VS, et al. Reproductive genetic counseling challenges associated with diagnostic exome sequencing in a large academic private reproductive genetic counseling practice. Prenat Diagn 2015;35:1022-1029.

17. Alamillo $\mathrm{CL}$, Powis $\mathrm{Z}$, Farwell $\mathrm{K}$, et al. Exome sequencing positively identified relevant alterations in more than half of cases with an indication of prenatal ultrasound anomalies. Prenat Diagn 2015;35: 1073-1078.

18. Manichaikul A, Mychaleckyj JC, Rich SS, Daly K, Sale M, Chen WM. Robust relationship inference in genome-wide association studies. Bioinformatics 2010;26:2867-2873.

19. Richards $S$, Aziz N, Bale $S$, et al. Standards and guidelines for the interpretation of sequence variants: a joint consensus recommendation of the American College of Medical Genetics and Genomics and the Association for Molecular Pathology. Genet Med 2015;17:405-424.

20. Green RC, Berg JS, Grody WW, et al. ACMG recommendations for reporting of incidental findings in clinical exome and genome sequencing. Genet Med 2013;15:565-574.

21. Posey JE, Rosenfeld JA, James RA, et al. Molecular diagnostic experience of whole-exome sequencing in adult patients. Genet Med 2016;18: 678-685.

22. Grandjean H, Larroque D, Levi S. The performance of routine ultrasonographic screening of pregnancies in the Eurofetus Study. Am J Obstet Gynecol 1999;181:446-454.

23. National Collaborating Centre for Women's and Children's Health. Antenatal Care: Routine Care for the Healthy Pregnant Woman. National Institute for Health and Clinical Excellence: Guidance. RCOG Press: London, 2008.

24. Winter RM, Crawfurd MdA, Meire HB, Mitchell N. Osteopathia striata with cranial sclerosis: highly variable expression within a family including cleft palate in two neonatal cases. Clin Genet 1980;18:462-474.

25. Vasiljevic A, Azzi C, Lacalm A, et al. Prenatal diagnosis of osteopathia striata with cranial sclerosis. Prenat Diagn 2015;35:302-304.

26. Wilson NJ, Hansen CD, Azkur D, et al. Recessive mutations in the gene encoding frizzled 6 cause twenty nail dystrophy-expanding the differential diagnosis for pachyonychia congenita. J Dermatol Sci 2013;70:58-60. 


\section{ORIGINAL RESEARCH ARTICLE}

27. De Marco P, Merello E, Rossi A, et al. FZD6 is a novel gene for human neural tube defects. Hum Mutat 2012;33:384-390.

28. Shamseldin HE, Tulbah M, Kurdi W, et al. Identification of embryonic lethal genes in humans by autozygosity mapping and exome sequencing in consanguineous families. Genome Biol 2015;16:116.

29. Slavotinek A, Kaylor J, Pierce $H$, et al. CRB2 mutations produce a phenotype resembling congenital nephrosis, Finnish type, with cerebral ventriculomegaly and raised alpha-fetoprotein. Am J Hum Genet 2015;96:162-169.

30. Lamont RE, Tan WH, Innes AM, et al. Expansion of phenotype and genotypic data in CRB2-related syndrome. Eur J Hum Genet 2016;24: 1436-1444.

31. Ebarasi $L$, Ashraf $S$, Bierzynska $A$, et al. Defects of CRB2 cause steroidresistant nephrotic syndrome. Am J Hum Genet 2015;96:153-161.
YATES et al | Whole-exome sequencing on deceased fetuses with ultrasound anomalies

32. Westerfield L, Darilek S, van den Veyver IB. Counseling challenges with variants of uncertain significance and incidental findings in prenatal genetic screening and diagnosis. J Clin Med 2014;3:1018-1032.

33. Lohmann K, Klein C. Next generation sequencing and the future of genetic diagnosis. Neurotherapeutics 2014;11:699-707.

34. Prenatal diagnostic testing for genetic disorders. Practice Bulletin No. 162. American College of Obstetricians and Gynecologists. Obstet Gynecol 2016;127:976-978.

35. Yu H, Zhang VW. Precision medicine for continuing phenotype expansion of human genetic diseases. Biomed Res Int 2015;2015:745043.

36. Landrum MJ, Lee JM, Benson $M$, et al. ClinVar: public archive of interpretations of clinically relevant variants. Nucleic Acids Res 2016;44: D862-D868.

37. Rehm HL, Berg JS, Brooks LD, et al. ClinGen-the Clinical Genome Resource. N Engl J Med 2015;372:2235-2242. 